\title{
An unusual presentation of acute deep vein thrombosis after the Moderna COVID-19 vaccine-a case report
}

\author{
Chandur Bhan ${ }^{1,2}$, Nimarta Bheesham ${ }^{1,2}$, Fnu Shakuntulla ${ }^{3}$, Monica Sharma ${ }^{2,4}$, Chenyu Sun ${ }^{1,2}$, \\ Mitchell Weinstein ${ }^{2,4}$
}

${ }^{1}$ Department of Internal Medicine, Amita Health Saint Joseph Hospital, Chicago, IL, USA; ${ }^{2}$ Section of General Medicine, Amita Health Saint Joseph Hospital, Chicago, IL, USA; ${ }^{3}$ Department of Internal Medicine, Section of General Medicine, OSF Heart of Mary Medical Center, Urbana, IL, USA; ${ }^{4}$ Department of Infectious Diseases, Amita Health Saint Joseph Hospital, Chicago, IL, USA

Correspondence to: Mitchell Weinstein, MD. AMITA Health Saint Joseph Hospital Chicago, 2900 N. Lake Shore Drive, Chicago 60657, IL, USA. Email: Mitchell.weinstein@amitahealth.org.

\begin{abstract}
Severe acute respiratory syndrome coronavirus 2 (SARS-CoV-2) has caused a worldwide pandemic of the multisystem disease coronavirus disease-2019 (COVID-19). Since the development of COVID-19 vaccines, there has been extensive monitoring for potential serious side effects. We report an unusual presentation of acute deep vein thrombosis (DVT) in the right upper extremity of a 27-year-old Caucasian female, 3 days after receipt of her second dose of the Moderna COVID-19 vaccine. Her relevant thrombophilia workup was negative on initial presentation. She was treated with rivaroxaban for 3 months and her symptoms of right upper extremity swelling, and pain improved. Considering our case did not have any evidence of thrombocytopenia, we discuss the possible pathophysiology of acute DVT following Moderna COVID-19 vaccine in contrast to adenoviral vector COVID-19 vaccines (ChAdOx1 nCoV-19 and Ad26.COV2.S), including mRNA COVID-19 vaccine binding to pattern recognition receptors (PRR) in the endosomes and cytosol leading to a pro inflammatory cascade and coagulopathy. We highlight the importance of initial workup for acute DVT post COVID-19 vaccination, that should include complete blood count (CBC) with platelet count, international normalized ratio (INR), prothrombin time (PTT), D-dimer levels, fibrinogen levels, platelet factor 4 (PF4)/heparin enzyme-linked immunosorbent assays (ELISA) followed by a confirmatory PF4 platelet activation assay such as serotonin release assay, P-selectin expression assay, or heparin induced platelet aggregation (HIPA) assay, and imaging for thrombosis.
\end{abstract}

Keywords: Coronavirus disease-2019 (COVID-19); vaccine; deep vein thrombosis (DVT); case report

Submitted Jun 03, 2021. Accepted for publication Jul 30, 2021.

doi: 10.21037/atm-21-2772

View this article at: https://dx.doi.org/10.21037/atm-21-2772

\section{Introduction}

Severe acute respiratory syndrome coronavirus 2 (SARS-CoV-2) has caused a worldwide pandemic of the multisystem disease, coronavirus disease-2019 (COVID-19). In December 2020, the United States Food and Drug Administration issued an Emergency Use Authorization (EUA) for the Moderna COVID-19 vaccine (mRNA1273 SARS-CoV-2) (1). The 2-dose vaccine had a reported vaccine efficacy of $94.1 \%$ in preventing illness in initial trials. It is generally well tolerated with mild side effects including local injection site reactions and systemic side effects (2). Venous thromboembolism has been rarely described among mRNA vaccines. We describe the uncommon adverse event of an acute deep venous thrombosis associated with the Moderna COVID-19 vaccine. We present the following article in accordance with the CARE reporting checklist (available at https://dx.doi. org/10.21037/atm-21-2772).

$\wedge$ ORCID: 0000-0003-3812-3164. 


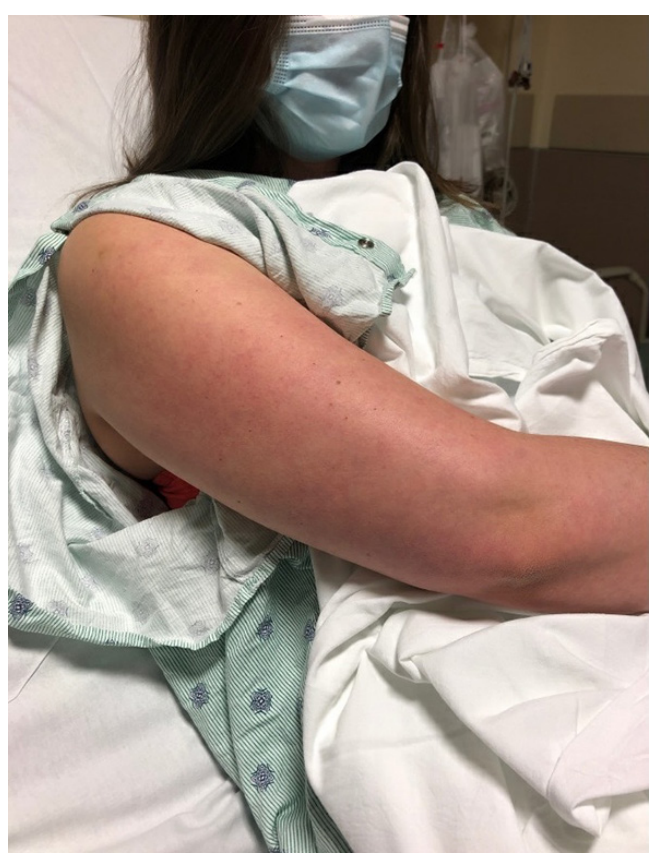

Figure 1 Right arm swelling and erythema.

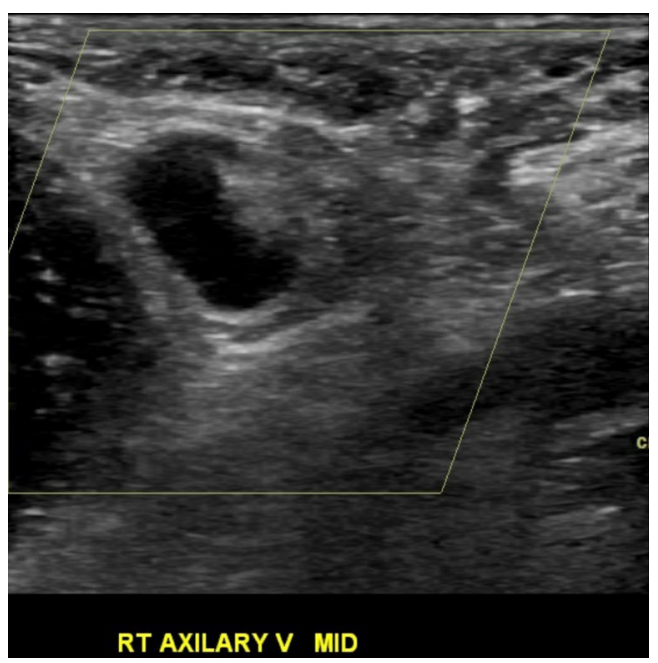

Figure 2 Ultrasound of the right upper extremity showing a thrombus in the right axillary vein.

\section{Case presentation}

A 27-year-old Caucasian female had been in her usual state of good health until the morning of hospital presentation when she developed right upper extremity generalized swelling, pain, and redness (Figure 1). She received the second dose of the Moderna COVID-19 vaccine to the right deltoid muscle 3 days before the onset of symptoms. Her first dose of the Moderna COVID-19 vaccine in her right deltoid muscle was 4 weeks previously and was well tolerated with only mild soreness at the injection site which resolved within a few days. Her past medical history and family history were unremarkable, including no prior blood clots, COVID-19 infection or smoking. She was never tested for any thrombophilic condition previously. She was not on any medications but had a hormonal intrauterine device (IUD) in place for 21 months prior to the current presentation.

On physical examination, she had generalized swelling of the right upper extremity from the hand to the shoulder area, with bruising and erythema around the injection site in the deltoid. Her initial laboratory results included an elevated D-dimer level $536 \mathrm{ng} / \mathrm{mL}$, an international normalized ratio (INR) 1.2 seconds, a prothrombin time (PTT) 13.5 seconds, and a platelet count 217,000 per cubic $\mathrm{mm}$. Venous duplex ultrasound showed an acute thrombosis involving the right subclavian and axillary veins (Figure 2). CT angiogram of the chest was negative for pulmonary embolism; however, it showed extensive bilateral axillary lymphadenopathy and mild left subclavian vein extrinsic compression between the clavicle and first rib. Subsequently, her thrombophilia workup was negative, including cardiolipin antibodies, beta-2 glycoprotein antibodies, factor 5 Leiden, prothrombin gene mutation, lupus anticoagulant panel, and Russell viper venom time. Heparin-induced thrombocytopenia platelet factor 4 (PF4) antibody was not checked. She was started on an intravenous unfractionated heparin infusion with activated partial thromboplastin time (aPTT) as a monitor for anticoagulation response, and was subsequently discharged on rivaroxaban for 3 months. At 2 weeks post discharge follow up, her arm swelling, and pain had significantly decreased. All procedures performed in studies involving human participants were in accordance with the ethical standards of the institutional and/or national research committee(s) and with the Helsinki Declaration (as revised in 2013). Written informed consent was obtained from the healthcare power of attorney of the patient for publication of this case report and accompanying images.

\section{Discussion}

Through the first month of the COVID-19 vaccination campaign (December 14, 2020-January 13, 2021), 13.7 million vaccine doses were administered (3). Ninety point eight 
percent of adverse events reported through the Vaccine Adverse Event Reporting System (VAERS) database were classified as nonserious (3). The symptoms most frequently reported by Moderna following the second dose of the vaccine were local pain $(88.4 \%)$, fatigue $(65.7 \%)$, headache (58.9\%), myalgia (58\%), chills (44.4\%), arthralgia (42.9\%), nausea/vomiting (19\%), fever (15.7\%), axillary swelling/ tenderness (14.2\%), local swelling (12.3\%) and local erythema (8.7\%) (2). No unexpected patterns of adverse reactions or other safety concerns were identified during early monitoring by the Centers for Disease Control and Prevention (CDC) (3).

Venous thromboembolism has rarely been reported following vaccination. In a study looking at the association of venous thromboembolism post influenza vaccine administration in a population above age 50 years, there was no increased risk overall. In a post-hoc analysis, an increased risk of venous thromboembolism within 10 days of influenza vaccination was found in current tobacco smokers (4). Research has also indicated a transient increase in proinflammatory cytokine production after influenza vaccination $(5,6)$ which theoretically could explain the association.

As of April 01, 2021, VAERS has collected at least 17 case reports of acute deep vein thrombosis (DVT) after the Moderna COVID-19 vaccine (7). Case reports have primarily involved acute DVTs of the lower extremities. The VAERS system, however, is subject to reporting bias and may include cases not directly caused by vaccination.

Our case of DVT post Moderna COVID-19 vaccine should not be confused with recent reported cases of vaccine-induced immune thrombotic thrombocytopenia (VITT). The latter condition has been associated with PF4 antibodies induced by adenoviral vector COVID-19 vaccines [ChAdOx1 nCoV-19 (8) and Ad26.COV2.S (9)]. This leads to thrombocytopenia and platelet activation with venous and arterial thromboses at multiple and unusual sites including cerebral venous sinus thromboses. Our case did not have thrombocytopenia and had localized venous thrombosis at the ipsilateral arm where the patient was vaccinated, presumably predominantly due to mechanical factors associated with soft tissue swelling, adenopathy, local venous compression and stasis. Our patient may have an underlying anatomic variant contributing to a partial compression of the subclavian vein between the first rib and the clavicle, which could have been further exacerbated by the soft tissue swelling and lymphadenopathy post vaccination. There has also been suggestion that mRNA
COVID-19 vaccine may bind to pattern recognition receptors (PRR) in the endosomes and cytosol leading to a pro inflammatory cascade and coagulopathy (10).

Although some oral contraceptives may increase the risk of VTE, previous studies have shown that hormonal IUD do not increase the risk significantly, odds ratio (OR) 0.3 ; [95\% confidence interval (CI), 0.1 to 1.1$]$ in one study (11) and adjusted rate ratio (aRR) of 0.89 ; (0.64 to 1.26 ) in another (12). The fact that our patient had her IUD in place for 21 months and the temporal association of her recent vaccination is more suggestive of a true association of the DVT and the Moderna COVID-19 vaccine rather than her any relationship to her IUD.

We acknowledge several limitations in conclusively attributing the DVT to vaccination, including the incomplete testing for PF4 antibodies and the fact that this a single case report rather than a larger scale population surveillance. Additional research is needed to better evaluate the prevalence and pathophysiology of thrombosis with the Moderna and other COVID-19 vaccines. In patients who present with significant arm swelling post vaccination, workup should include complete blood count (CBC) with platelet count, INR, PTT, D-dimer levels, fibrinogen levels, PF4/heparin enzyme-linked immunosorbent assays (ELISA) followed by a confirmatory $\mathrm{PF} 4$ platelet activation assay such as serotonin release assay, $\mathrm{P}$-selectin expression assay, or heparin induced platelet aggregation (HIPA) assay; and imaging for thrombosis (13). While rare, our report should serve as an alert for an uncommon complication of the Moderna COVID-19 vaccine and remind that not all post COVID vaccination limb swelling should be disregarded.

\section{Acknowledgments}

Funding: None.

\section{Footnote}

Reporting Checklist: The authors have completed the CARE reporting checklist. Available at https://dx.doi. org/10.21037/atm-21-2772

Conflicts of Interest: All authors have completed the ICMJE uniform disclosure form (available at https://dx.doi. org/10.21037/atm-21-2772). The authors have no conflicts of interest to declare.

Ethical Statement: The authors are accountable for all 
aspects of the work in ensuring that questions related to the accuracy or integrity of any part of the work are appropriately investigated and resolved. All procedures performed in studies involving human participants were in accordance with the ethical standards of the institutional and/or national research committee(s) and with the Helsinki Declaration (as revised in 2013). Written informed consent was obtained from the healthcare power of attorney of the patient for publication of this case report and accompanying images. A copy of the written consent is available for review by the editorial office of this journal.

Open Access Statement: This is an Open Access article distributed in accordance with the Creative Commons Attribution-NonCommercial-NoDerivs 4.0 International License (CC BY-NC-ND 4.0), which permits the noncommercial replication and distribution of the article with the strict proviso that no changes or edits are made and the original work is properly cited (including links to both the formal publication through the relevant DOI and the license). See: https://creativecommons.org/licenses/by-nc-nd/4.0/.

\section{References}

1. U.S. Food \& Drug Administration. FDA Takes Additional Action in Fight Against COVID-19 By Issuing Emergency Use Authorization for Second COVID-19 Vaccine. 2020 [cited 2021 Apr 1]. Available online: https://www.fda.gov/ news-events/press-announcements/fda-takes-additionalaction-fight-against-covid-19-issuing-emergency-useauthorization-second-covid

2. Baden LR, El Sahly HM, Essink B, et al. Efficacy and Safety of the mRNA-1273 SARS-CoV-2 Vaccine. N Engl J Med 2021;384:403-16.

3. Gee J, Marquez P, Su J, et al. First month of COVID-19 vaccine safety monitoring - United States, December 14, 2020-January 13, 2021. MMWR Morb Mortal Wkly Rep 2021;70:283-8.

Cite this article as: Bhan C, Bheesham N, Shakuntulla F, Sharma M, Sun C, Weinstein M. An unusual presentation of acute deep vein thrombosis after the Moderna COVID-19 vaccine-a case report. Ann Transl Med 2021;9(20):1605. doi: $10.21037 / \mathrm{atm}-21-2772$
4. Vickers ER, McClure DL, Naleway AL, et al. Risk of venous thromboembolism following influenza vaccination in adults aged 50 years and older in the Vaccine Safety Datalink. Vaccine 2017;35:5872-7.

5. Tsai MY, Hanson NQ, Straka RJ, et al. Effect of influenza vaccine on markers of inflammation and lipid profile. J Lab Clin Med 2005;145:323-7.

6. Christian LM, Iams JD, Porter K, et al. Inflammatory responses to trivalent influenza virus vaccine among pregnant women. Vaccine 2011;29:8982-7.

7. Centers for Disease Control and Prevention(CDC). The Vaccine Adverse Event Reporting System (VAERS). Available online: http://wonder.cdc.gov/vaers.html (Accessed on Apr 1, 2021).

8. Greinacher A, Thiele T, Warkentin TE, et al. Thrombotic Thrombocytopenia after ChAdOx1 nCov-19 Vaccination. N Engl J Med 2021;384:2092-101.

9. See I, Su JR, Lale A, et al. US Case Reports of Cerebral Venous Sinus Thrombosis With Thrombocytopenia After Ad26.COV2.S Vaccination, March 2 to April 21, 2021. JAMA 2021;325:2448-56.

10. Talotta R. Do COVID-19 RNA-based vaccines put at risk of immune-mediated diseases? In reply to "potential antigenic cross-reactivity between SARS-CoV-2 and human tissue with a possible link to an increase in autoimmune diseases". Clin Immunol 2021;224:108665.

11. van Hylckama Vlieg A, Helmerhorst FM, Rosendaal FR. The risk of deep venous thrombosis associated with injectable depot-medroxyprogesterone acetate contraceptives or a levonorgestrel intrauterine device. Arterioscler Thromb Vasc Biol 2010;30:2297-300.

12. Lidegaard Ø, Løkkegaard E, Svendsen AL, et al. Hormonal contraception and risk of venous thromboembolism: national follow-up study. BMJ 2009;339:b2890.

13. Kantarcioglu B, Iqbal O, Walenga JM, et al. An Update on the Pathogenesis of COVID-19 and the Reportedly Rare Thrombotic Events Following Vaccination. Clin Appl Thromb Hemost 2021;27:10760296211021498. 